\title{
Hereditäre Tumorerkrankungen des Gastrointestinaltrakts
}

\author{
Gabriela Möslein $^{\mathrm{a}} \quad$ Andrea Tannapfel ${ }^{\mathrm{b}}$

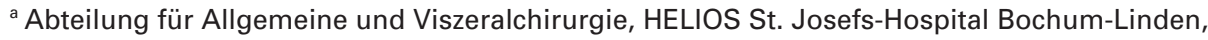 \\ ${ }^{\mathrm{b}}$ Institut für Pathologie, Ruhr-Universität Bochum, Deutschland
}

Maligne Tumoren des Kolons und des Rektums entstehen zu etwa $70-80 \%$ sporadisch, d.h. ohne erkennbaren Grund und typischerweise in einem höheren Lebensalter. Etwa 3-5\% der Karzinome beruhen auf einer genetischen Prädisposition, wobei in der Mehrzahl ein autosomal dominanter Erbgang vorliegt. Hiervon zu unterscheiden sind die heute ebenfalls unterschätzten, zu etwa $20 \%$ vorliegenden familiären Prädispositionen, bei denen rezessive Erbgänge oder modifizierende genetische Faktoren eine Rolle spielen. Die diagnostisch wegweisenden und zunehmend erschwinglichen Möglichkeiten der molekulargenetischen Diagnostik bleiben aufgrund fehlender klinischer Beachtung viel zu oft ungenutzt.

Die vorliegende zusammenfassende Darstellung hereditärer Tumorerkrankungen in der Viszeralmedizin beschäftigt sich mit den bisher bekannten klinisch und/oder molekulargenetisch charakterisierten kolorektalen Prädispositionssyndromen. Sowohl das häufige Lynch-Syndrom und seine Abgrenzung zu HNPCC (Hereditary Non-Polyposis Colorectal Cancer) als auch die deutlich selteneren Erkrankungen, wie die familiäre adenomatöse Polyposis (FAP), das Peutz-JeghersSyndrom (PJS) und weitere Polyposis-Syndrome, werden aus Sicht der klinischen - einschließlich der (chemo-)präventiven und prophylaktisch-therapeutischen -, der pathologischen sowie der molekular- und humangenetischen Standards aktualisiert. Es ist gelungen, namhafte, international anerkannte Experten für die jeweiligen Entitäten zu gewinnen. Wichtig in diesem Zusammenhang ist den beiden Herausgeberinnen, zu einer gesteigerten «Awareness» für die weit unterdiagnostizierten Risikopersonen aus diesem Formenkreis zu sensibilisieren. Wann sollte man eine weitergehende humangenetische oder klinische Beratung veranlassen - dabei spielt sowohl die Eigen- als auch die Familienanamnese von Patienten eine große Rolle. Wer hat die Gesamtanzahl metachron auftretender Polypen bei Patienten im Blickfeld? Die MallorcaGruppe (www.mallorca-group.eu) definiert 20 Polypen jedweder Histologie bis zu einem Alter von 60 Jahren als abklärungsbedürftig («Mallorca Polyposis»). Jedoch ist auch die «Awareness» der Pathologen bei der histologischen Begutachtung von Tumoren mit spezifischen histologischen Charakteristika gefragt. Die Frage steht im Raum, ob eine routinemäßige Mikrosatellitenuntersuchung bei allen kolorektalen Tumoren (immunhistochemisch) zum Standard gehören sollte.

Wegen des syndromalen Charakters der disponierenden Entitäten wurde besonderer Wert auf den oberen Gastrointestinaltrakt und extrakolonische Begleiterkrankungen wie z.B. Fibromatosen gelegt. Alle Autoren haben den aktuellen Stand der Literatur prägnant dargestellt und auch neueste Empfehlungen aus noch nicht publizierten Studien thematisiert. Somit stellt das vorliegende Heft eine hochaktuelle «State of the Art»-Lektüre zu den hereditären kolorektalen Karzinomen dar.

\section{KARGER \\ Fax +497614520714 \\ Information@Karger.de}

www.karger.com
(C) 2011 S. Karger GmbH, Freiburg

1662-6664/11/0274-0264\$38.00/0

Accessible online at:

www.karger.com/vim
Prof. Dr. med. Gabriela Möslein

Abteilung für Allgemeine und Viszeralchirurgie

HELIOS St. Josefs-Hospital Bochum-Linden

Axstraße 35, 44879 Bochum, Deutschland

Tel. +49234 418-252, Fax -862

gabriela.moeslein@helios-kliniken.de 